\title{
Coronae of Cool Stars
}

\author{
M. Audard ${ }^{1}$, S. A. Drake ${ }^{2,3}$, M. Güdel ${ }^{4}$, R. $\mathrm{Mewe}^{5}$, R. Pallavicini ${ }^{6}$, \\ T. Simon ${ }^{7}$, K. P. Singh ${ }^{8}$, S. L. Skinner ${ }^{9}$, N. E. White ${ }^{3}$ \\ ${ }^{1}$ Columbia University, ${ }^{2}$ USRA, ${ }^{3}$ NASA/GSFC, ${ }^{4}$ Paul Scherrer Institut, \\ ${ }^{5}$ SRON-Utrecht, ${ }^{6}$ Palermo Observatory, ${ }^{7}$ University of Hawaii, ${ }^{8}$ Tata \\ Institute of Fundamental Research, ${ }^{9}$ CASA/Univ. of Colorado
}

\begin{abstract}
We present preliminary results of grating observations of YY Mensae and V824 Arae by Chandra and XMM-Newton. Spectral features are presented in the context of the emission measure distributions, the coronal abundances, and plasma electron densities. In particular, we observe a coronal N/C enhancement in YY Men believed to reflect the photospheric composition (CN cycle). Finally, we interpret line broadening in YY Men as Doppler thermal broadening in its very hot corona.
\end{abstract}

\section{Introduction}

Grating spectrometers on board Chandra and XMM-Newton have revolutionized the field of X-ray spectroscopy of cosmic plasmas. Individual lines can be measured in the soft X-ray energy range with sufficient accuracy to permit measurements of elemental abundances, plasma densities, or simply to reveal the spectroscopic nature of a cosmic plasma (e.g., see Paerels \& Kahn 2003 for a review on high-resolution X-ray spectroscopy with Chandra and XMM-Newton).

Many cool stars are bright X-ray sources thanks to their hot coronae and their close distances. They are ideal targets for X-ray spectroscopy since they display a wealth of bright emission lines of various elements. Recent reviews summarize results obtained in the first years after the launch of Chandra and XMM-Newton (e.g., Audard 2003, Güdel 2003, Linsky 2003). In particular, patterns in coronal abundances have been recognized in which elements with a first ionization potential (FIP) $<10 \mathrm{eV}$ are underabundant with respect to the high-FIP elements when observed in very active stars; this is opposite to the solar FIP effect in which low-FIP elements are overabundant whereas high-FIP elements are of photospheric composition. However, coronal abundances in a sample of stars with a broad range of magnetic activity seem to reverse from a solar-like FIP effect in the least active, old stars to an "inverse"-FIP effect in the most active, young stars (Güdel et al. 2002). The trend is suggestive and a larger sample is certainly needed.

Two very active stars, YY Men and V824 Ara, have been observed with grating spectrometers on board Chandra and XMM-Newton in order to study, in particular, their coronal abundances. YY Men is one of the (rare) class of single giants with very hot coronae, whereas V824 Ara is an example of a close main-sequence, very active $\mathrm{RS} C \mathrm{CV}$ binary. We present here preliminary results of the grating observations. 


\section{Observations and Data Reduction}

Chandra observed YY Men in the ACIS-S/HETG configuration for $74 \mathrm{ks}$ in February 1-2, 2002, whereas the 86 ks-long XMM-Newton observation dates from October 5-6, 2001. The RS CVn binary V824 Ara was observed with Chandra ACIS-S/HETGS for 94 ks on July 10-11, 2002. The Chandra data sets were reduced with CIAO 2.3 with CALDB 2.21 using standard techniques described in threads; however, events for V824 Ara and LDS 587B were resolved using narrower, non-standard grating arms (10 times the $1 \sigma$ cross-dispersion width instead of the default 35 times). Thanks to the low background in ACIS, no background spectra were used in the Chandra spectral analysis allowing us to use the robust $\mathrm{C}$ statistics. The XMM-Newton grating data were reduced with SAS 5.4.1 using standard techniques (see Audard et al. 2003).

\section{3. $\quad$ YY Mensae $=$ HD 32918}

The Chandra and XMM-Newton observations were motivated by the very hot temperature detected with ROSAT PSPC and ASCA (Güdel et al. 1996) and its extreme luminosity $\left(\log L_{\mathrm{X}} \sim 32\right.$ for a distance of $\left.291 \mathrm{pc}\right)$. YY Men $(P \sim 9.55 \mathrm{~d}$; $v \sin i \sim 50 \mathrm{~km} \mathrm{~s}^{-1}$; e.g., Collier Cameron 1982) is a member of a looselydefined class of rapidly rotating single G and K giant stars ("FK Com stars"), whose outstanding property is a projected equatorial velocity measured up to $160 \mathrm{~km} \mathrm{~s}^{-1}$, in contrast with the expected maximum of $6 \mathrm{~km} \mathrm{~s}^{-1}$ for giants. One of the leading theories to explain the extreme properties of FK Com stars suggests that they were formed by coalescence of a contact binary when one of the components entered into the giant stage (Bopp \& Stencel 1981).

Figure 1 shows the Chandra ACIS-S/HETG first-order HEG and MEG spectra. Several emission lines are detected; in particular $\mathrm{Fe}$ lines in various ionization states can be found, from Fe XVII to Fe XXV, indicating a broad distribution of plasma temperatures, typically from $4 \mathrm{MK}$ to $30 \mathrm{MK}$. Elements such as $\mathrm{N}, \mathrm{O}, \mathrm{Ne}, \mathrm{Mg}, \mathrm{Si}, \mathrm{S}, \mathrm{Ar}$, and $\mathrm{Ca}$ are detected as well, mostly in $\mathrm{H}$-like and He-like transitions. The strong Ly $\alpha$ lines indicated a dominant very hot plasma, also suggested by the $\mathrm{Fe} \mathrm{K} \alpha$ complex and the strong underlying continuum.

We used a multi- $T$ approach and fitted the MEG and HEG data simultaneously to obtain a discretized emission measure (EM) distribution of YY Men along with coronal abundances. We also approached the data differently and extracted line fluxes to construct a continuous EM distribution and obtain coronal abundances as well. We describe our methodology elsewhere (Audard et al. 2004). We report here, in brief, on the 3-T approach: a very hot (40 MK) plasma dominates the X-ray spectrum; however two other plasma components are detected as well ( $7 \mathrm{MK}$ and $14 \mathrm{MK}$ ). No lower $T$ component could be detected either in the Chandra HETGS data or in the RGS spectrum. The EM ratios are 1:2:19 (where the coolest component has $\mathrm{EM}=1$ ), the X-ray luminosity is $L_{\mathrm{X}}=2.2 \times 10^{32} \mathrm{erg} \mathrm{s}^{-1}(0.1-10 \mathrm{keV})$ with $N_{\mathrm{H}}=7.5 \times 10^{20} \mathrm{~cm}^{-2}$. Coronal abundances indicate a pattern reminiscent of the inverse FIP effect observed in active RS CVn binaries (e.g., Audard et al. 2003). However, a high N abundance $([\mathrm{N} / \mathrm{H}]=+0.3)$ probably reflects a high photospheric $\mathrm{N}$ abundance due to $\mathrm{CN}$ processing in the stellar interior; indeed the RGS spectrum does not show a $\mathrm{C}$ VI Ly $\alpha$ line, indicating a low $[\mathrm{C} / \mathrm{N}]=-0.8$ ratio. 

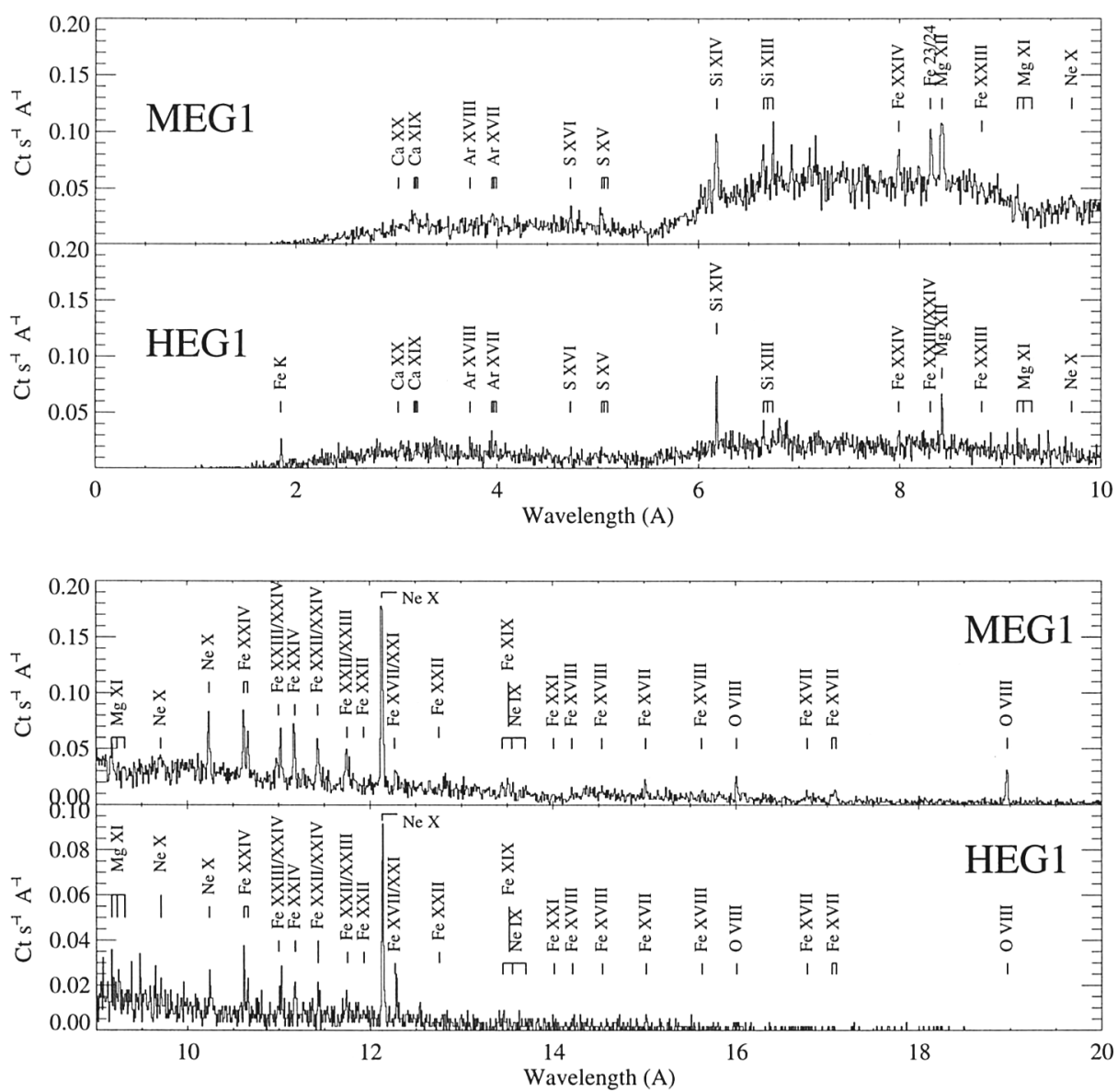

Figure 1. Chandra ACIS-S/HETGS spectrum of YY Men. Several emission lines are labeled. Despite the strong photoelectric absorption and the small effective area at $25 \AA\left(5 \mathrm{~cm}^{2}\right)$, a clear N vil Ly $\alpha$ line is detected (not shown here).

Line broadening is detected in the Ne x Ly $\alpha$ line $(\sigma=7 \pm 0.7 \mathrm{~m} \AA$; Figure 2). Similar tentative detections are reported in $\operatorname{Ne} \mathrm{x} \operatorname{Ly} \beta, \mathrm{O}$ VIII Ly $\alpha, \operatorname{Mg}$ XII Ly $\alpha$, and Si XIV Ly $\alpha$. However, S, Ar, and Ca Ly $\alpha$ lines are either too weak or at too short wavelength to detect line broadening. N VII Ly $\alpha$, however, does not show evidence of broadening. Th Ne $\mathrm{x}$ line broadening is unlikely to be due to rotation since (i) it would require $v \sin i \gg 50 \mathrm{~km} \mathrm{~s}^{-1}$, and (ii) it would be easily detected in the longer wavelength lines. Thus we interpret the observed broadening as Doppler thermal broadening in the very hot corona of YY Men. The $\mathrm{N}$ VII line, despite the lower atomic mass of $\mathrm{N}$ than of $\mathrm{Ne}$, is probably not broadened because it is mostly formed by the lower $T$ components, while the Ne lines are mostly formed by the very hot plasma (as are the lines with tentative detections of line broadening). This is also supported by the absence 

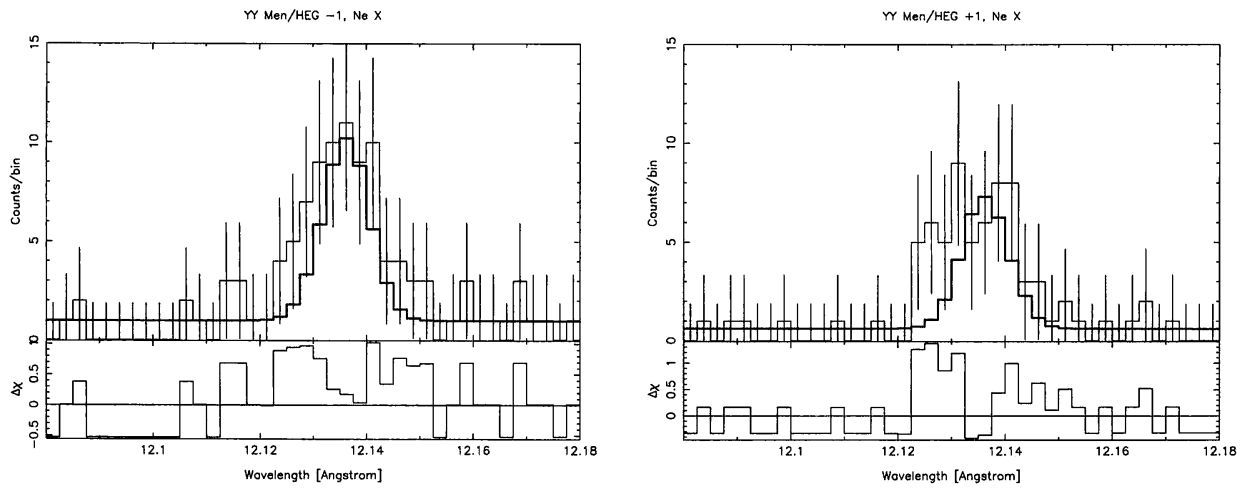

Figure 2. HEG -1 (left) and +1 (right) spectra of YY Men around the Ne $\mathrm{x}$ Ly $\alpha$ line. The instrumental profile is overlaid, emphasizing the line broadening (probably due to Doppler broadening). Similar results are tentatively reported for other lines (see text).

of line broadening in Fe lines, probably due to the larger Fe atomic mass. The observed Doppler width $\left(\sigma_{\mathrm{D}}=(\lambda / c) \times \sqrt{k T / m}\right)$ corresponds to a plasma $T$ in the range $60-90 \mathrm{MK}$. Possibly additional broadenings, e.g., due to rotation, contribute to the total line width, which would lower the $T$ range.

\section{V824 Arae $=$ HD 155555}

The nearby $(31 \mathrm{pc})$ binary consists of G5 V+K0 V stars tidally locked with an orbital period of $1.68 \mathrm{~d}$. Based on its kinematics and its high $\mathrm{Li}$ abundance, V824 Ara is either in the pre-main-sequence phase (Pasquini et al. 1991) or in the zero-age-main-sequence phase (Martín \& Brandner 1995), with an age of $10^{7}-10^{8}$ yr. Its common proper-motion dM4.5e companion (33" away), LDS 587B, also displays high chromospheric and coronal activity. Since V824 Ara is so young, its photosphere should have near-solar photospheric composition.

Figure 3 shows the Chandra ACIS-S/HETG MEG spectrum of V824 Ara together with a best-fit 4- $T$ plasma model. The EM distribution differs from YY Men; it is flatter with $T$ of $3.7,7.7,14$, and $28 \mathrm{MK}, \mathrm{EM}$ ratios of 1:3:2:2.7, and $L_{\mathrm{X}}=4.4 \times 10^{30} \mathrm{erg} \mathrm{s}^{-1}(0.1-10 \mathrm{keV})$. The coronal abundances show a distinct inverse FIP effect $([\mathrm{Fe} / \mathrm{H}]=-0.5,[\mathrm{O} / \mathrm{H}]=-0.3,[\mathrm{Ne} / \mathrm{H}]=-0.1)$, except at very low FIP $(<7 \mathrm{eV})$, where the $\mathrm{Al}$ and $\mathrm{Ca}$ abundances seem to increase. A similar pattern was observed in the coronal abundances of the RS CVn binary $\sigma^{2} \mathrm{CrB}$ (Osten et al. 2003). The LDS 587B companion was sufficiently bright $\left(L_{\mathrm{X}}=\right.$ $2.8 \times 10^{29} \mathrm{erg} \mathrm{s}^{-1}$ ) and spatially resolved to produce a distinct grating spectrum that reveals a similar $T$ structure (although EM ratios for a 3-T fit are 1:1.1:1.6). Preliminary coronal abundances indicate no obvious correlation with the FIP.

Plasma densities can be derived from line ratios, typically from the forbidden and intercombination lines of He-like transitions. The well-developed HETGS spectrum of V824 Ara reveals no variation from the low density limit in the various He-like triplets, suggesting that the dominant X-ray emitting plasma has an electron density below $10^{10} \mathrm{~cm}^{-3}$. Since coronae are inhomogeneous, this result does not mean that no high-density plasma exists. However, it suggests that such plasma has a very low volume (since $E M \sim n_{e}^{2} V$ ). 

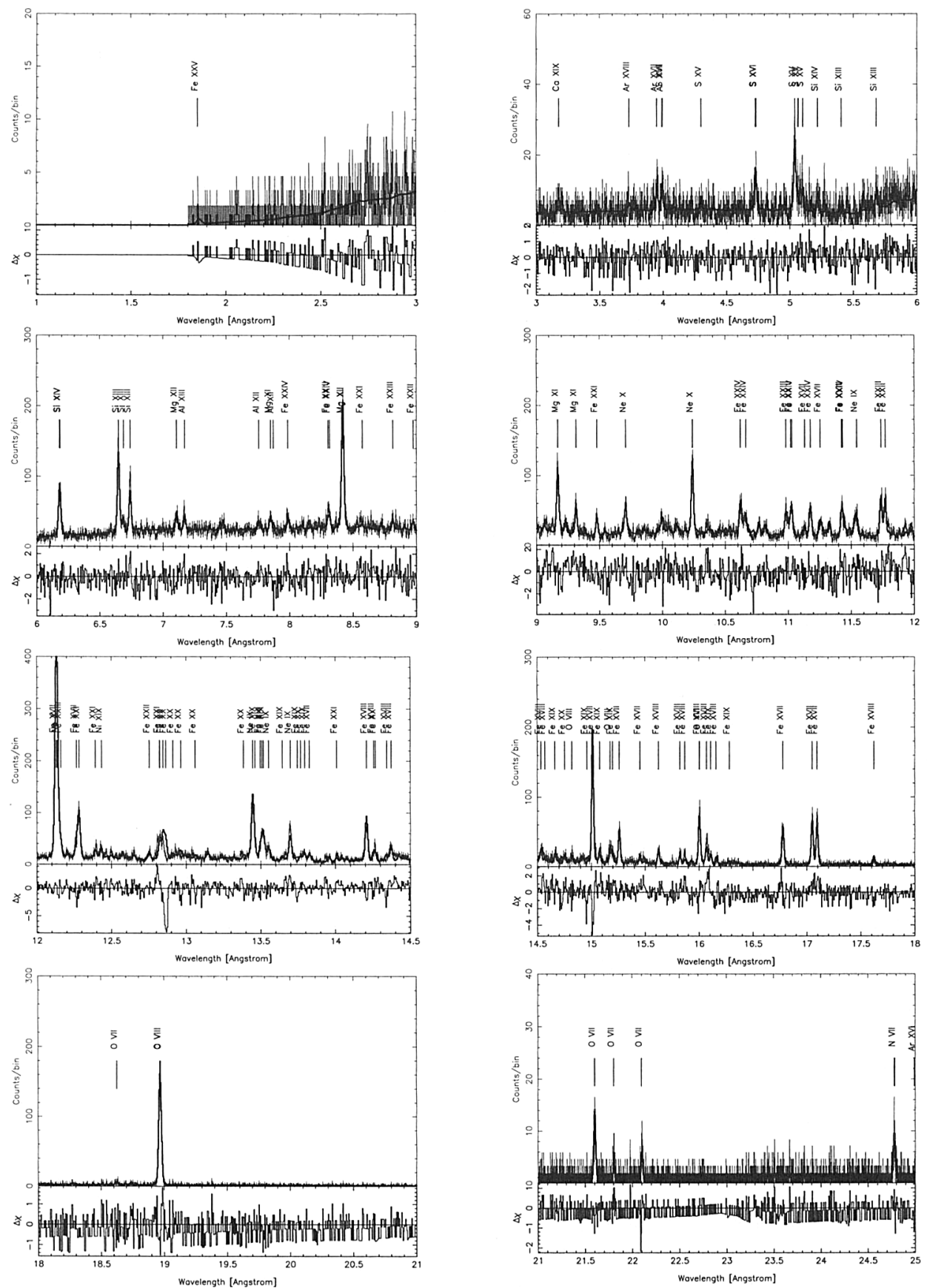

Figure 3. Chandra ACIS-S/HETG spectrum of V824 Ara. A 4-T best-fit model is overlaid. Residuals are shown in the lower panels. The bad fit of the Fe xx blend $(12.8 \AA)$ can be explained by inaccurate wavelengths estimated from lab measurements (Brown et al. 2002, Fig. 3b) listed in the APEC 1.3 database. 


\section{Conclusions}

We have presented results of observations of YY Men and V824 Ara with Chandra and XMM-Newton. The highly active stars show contrasting X-ray spectra: the EM distribution of YY Men is dominated by a very hot (40 MK) plasma but has measurable EM between 6 and $15 \mathrm{MK}$, whereas the binary V824 Ara displays a rather flat EM distribution from $3 \mathrm{MK}$ to $30 \mathrm{MK}$. Despite their differences, their coronal abundances (relative to the solar photospheric composition) generally follow the inverse FIP effect observed in other active binaries. However, coronal abundances in the M dwarf LDS 587B do not correlate with the FIP. The coronal $\mathrm{N}$ abundance in YY Men is enhanced (C is depleted), probably reflecting a true photospheric $\mathrm{N} / \mathrm{C}$ enhancement due to mixing of $\mathrm{CN}$-cycle material in the stellar interior. YY Men also shows line broadening in Ne x Ly $\alpha$ and possibly in other lines, which we interpret as Doppler broadening.

The general picture is that coronal abundances in very active stars display an inverse FIP effect, while inactive stars show a solar-like FIP effect (e.g., Güdel et al. 2002). However, most current studies are limited to comparing stellar coronal abundances to solar photospheric abundances because of the lack of accurate photospheric measurements in active stars. Thus caution is in effect, since coronal abundances could also be affected by anomalous photospheric compositions as shown here for YY Men, apart from selective, FIP-dependent coronal enrichment. Improvements in the photospheric composition in stars will help better understand the coronal composition.

Acknowledgments. We acknowledge support from the Swiss National Science Foundation (fellowship 81EZ-67388 \& grant 2000-058827), from NASA to Columbia University for XMM-Newton mission support and data analysis, and from SAO grant G02-3016X.

\section{References}

Audard, M. 2003, Adv. Space Res., 32, 927

Audard, M., Güdel, M., Sres, A., et al. 2003, A\&A, 398, 1137

Audard, M., et al. 2004, ApJ, in preparation

Bopp, B. W. \& Stencel, R. E. 1981, ApJ, 247, L131

Brown, G. V., Beiersdorfer, P., Liedahl, D. A., et al. 2002, ApJS, 140, 589

Collier Cameron, A. 1982, MNRAS, 200, 489

Güdel, M., Guinan, E. F., Skinner, S. L., \& Linsky, J. L. 1996, Röntgenstrahlung from the Universe, 33

Güdel, M. 2003, Adv. Space Res., in press

Güdel, M., Audard, M., Sres, A., et al. 2002, in $35^{\text {th }}$ ESLAB Symposium, ASP Conf. Ser. 277, eds. F. Favata \& J. J. Drake (San Francisco: ASP), 497

Linsky, J. L. 2003, Adv. Space Res., 32, 917

Martín, E. L. \& Brandner, W. 1995, A\&A, 294, 744

Osten, R. A., Ayres, T. R., Brown, A.,et al. 2003, ApJ, 582, 1073

Paerels, F. B. S., \& Kahn, S. M. 2003, ARA\&A, 41, 291

Pasquini, L., Cutispoto, G., Gratton, R., \& Mayor, M. 1991, A\&A, 248, 72 